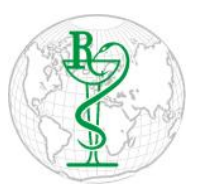

INDO GLOBAL JOURNAL OF

PHARMACEUTICAL SCIENCES

ISSN 2249- 1023

\title{
Prescription Completeness, Rational Prescribing, and Dispensing Practice at Referral Hospital, Ethiopia
}

\author{
Bezie Kebede ${ }^{1 *}$, Biset Asredaw ${ }^{2}$ \\ ${ }^{1}$ Department of Pharmacy, College of Medicine and Health Science, Mizan-Tepi University, Mizan-Teferi, Ethiopia \\ ${ }^{2}$ Department of Pharmacy, College of Medicine and Health Sciences, Bahir Dar University, Bahirdar, Ethiopia
}

Address for Correspondence: Bezie Kebede, beza.kebede21@gmail.com ; biset2006me@gmail.com

\begin{abstract}
Received:
14.08.2019

Accepted:

28.05.2020

Published:

15.12.2020

Keywords

Rational

prescribing;

Prescription

completeness;

Practice,

dispensing.
\end{abstract}

\begin{abstract}
Background: Rational drug use is a tool through which safe, effective and cost-effective medication is provided. Rational drug prescribing and dispensing ensures adherence to treatment and protects drug consumer from unnecessary drug exposure and bad consequences. Objective: To assess completeness of prescription and rational drug prescribing and dispensing practice and patient knowledge about their medication. Method: A prospective quantitative cross-sectional study was conducted in outpatient pharmacy both patients and dispensed drugs. Prescriptions which were dispensed from January 10, 2019 to May 10, 2019 were selected by systematic sampling technique for prescriber indicators analysis and patients interviewed for patient care indicator analysis. Results: Six hundred sixty-nine prescriptions were included in the study. The mean number of drugs per prescription was 1.46. Prescriptions dispensed with less than two drugs per prescription were $85.05 \%$. Antibiotics were the most prescribed drugs, $42.02 \%$ and less frequently prescribed drugs were injection $0.73 \%$. The weight of the patient was ignored in all prescriptions and only $13 \%$ of the prescription showed the diagnosis of the patient. The strength and duration of the drug were clearly stated in $81.89 \%, 74.61 \%$ of the prescriptions respectively. Only $68.03 \%$ drugs adequately labeled. Prescriptions with the name of the prescribers and dispensers were $32.74 \%$ and $20.93 \%$ respectively. Regarding knowledge of the patient, only $88.89 \%$ of patients interviewed had adequate knowledge of how to take the medication prescribed. The average counseling and dispensing time were $1 \mathrm{~min} 20 \mathrm{sec}$ and $11 \mathrm{~min} 54$ sec respectively. Conclusion: Most of the prescriptions were incomplete. Majority of prescriptions lack important patient information. So there is a need for managerial and educational intervention to improve rational drug prescribing and dispensing practices. () 2020 iGlobal Research and Publishing Foundation. All rights reserved.
\end{abstract}

Cite this article as: Kebede, B.; Asredaw, B. Prescription completeness, rational prescribing, and dispensing practice at referral hospital, Ethiopia. Indo Global J. Pharm. Sci., 2020; 10(4): 39-45. DOI: http://doi.org/10.35652/IGJPS.2020.10407.

\section{INTRODUCTION}

Rational use of drugs requires that patients receive medications appropriate to their clinical needs, in terms of doses that can meet individual requirements for an adequate duration of treatment, at the possible lowest cost and most effective drugs [1]. According to World Health Organization (WHO) report, irrational drug use is a significant problem for the community [2].

Medicines play an important role in healthcare delivery system such as disease prevention, prophylaxis, vaccination, for diagnosis and treatment. Availability and affordability of good quality drugs along with rational use is paramount for effective health care delivery and to improve quality of life of the society. However, irrational drug use is very prevalent, especially in developing countries due to irrational prescribing, dispensing, medication administration and patient use. Prescribing practice indicators measure the performance of healthcare providers in several key dimensions related to the appropriate use of drugs. Rational drug use is a tool through which safe, effective and cost-effective medication is provided. It can be improved by the collaborated efforts of prescribers, dispensers and drug consumers [1]. 


\section{Indo Global Journal of Pharmaceutical Sciences, 2020; 10(4): 39-45}

Irrational drug use is characterized by the use of drugs when no pharmacologic therapy is indicated, the use of ineffective drugs for specific condition requiring drug therapy, the use of drugs which is contraindicated without considering individualization, the use of drugs of uncertain safety status, failure to provide availability of safe and effective drugs, the use of too many medicines per patient (3). Inappropriate use of antibiotics, often inadequate dosage, antibiotics prescribing for non-bacterial infections, the use of correct drugs with incorrect administration, techniques including medication reconstitution. There is also irrational drug use in which over use of injections when oral formulations can be more appropriate and failure to prescribe in accordance with clinical Guidelines [4, 5]. Irrational use of medicines can stimulate excessive medication demand by the patient $[6,7]$.

Patient care indicators such as average consultation time, average dispensing time, percentage of patients 'knowledge of correct dosage which understand the way how drugs are used. It is important to consider what takes place at health facilities from both the provider's and the patient's perspective. The patient care indicator address key aspects of what patients experience at health facilities, and how well they have been prepared to deal with the pharmaceuticals that have been prescribed and dispensed [8]. As different literatures showed that irrational drug use is prevalent and causes patient harm.

Therefore, this drug use evaluation will be valuable to determine how much irrational drug use is prevalent and assess completeness of prescription in this referral hospital.

\section{MATERIALS AND METHODS}

\section{Study Area and Design}

The study was conducted at Felege Hiwot Referral Hospital (FHRH), Bahir dar, Ethiopia. FHRH is located in Bahir dar town which is $565 \mathrm{~km}$ far from Addis Ababa and it is found northwest Ethiopia. It is one of the largest government hospitals in Ethiopia. FHRH is offering diagnosis and treatment for approximately 18,465 patients per month. There are about 5 pharmacies within the hospital. This evaluation is conducted at outpatient pharmacy from January 10, 2019 May 10, 2019.

A prospective cross-sectional study was employed in the hospital by selected sample prescription from outpatient pharmacy of the hospital for three month period. All patients who have prescription in outpatient pharmacy which were dispensed during the data collection period were as a source of sample to assess rational drug use and completeness of prescription as well as the prescribing and dispensing practices at the hospital. Patients who came to the outpatient pharmacy were interviewed to evaluate patient care indicators. Data for computation of core prescribing and dispensing were collected as per the recommendations of WHO guideline [9].

\section{Data Collection Tool and Procedures}

Data was collected by four pharmacy personnel who are not working in FHRH after two day training concerning how to collect data by using WHO standard data collection formats of prescribing indicators, patient care indicators and facility indicator. The data on prescribing indicators were collected using prescriptions, and for facility indicators observing the availability of standard treatment guidelines and formularies in the hospitals and taking the hospitals yearly data on availability of tracer drugs and for patient care indicators by observing and asking closed ended questions directly the patients with their dispensed drugs prospectively.

\section{Data Analysis}

All data in the ordinary prescription and patient care indicator recorded was cleaned and entered in to Epi data 3.1. All the required statistical analysis was carried out with SPSS version 21 . The result was summarized with frequencies, percentages, graphs and tables.

Table 1: Summary of Prescription Paper Issued With Patient Related Information at FHRH January 10, 2019May 10, 2019

\begin{tabular}{|c|c|c|c|}
\hline \multicolumn{4}{|c|}{ Standard prescription paper used = 669 } \\
\hline \multicolumn{4}{|c|}{ Patient information } \\
\hline Indicators & Number(n) & Percentage & Gold standard \\
\hline Name of patient & 667 & $99.70 \%$ & $100 \%$ \\
\hline Age & 619 & $92.53 \%$ & $100 \%$ \\
\hline Sex & 616 & $92.08 \%$ & $100 \%$ \\
\hline Address & 0 & $0 \%$ & $100 \%$ \\
\hline Diagnosis & 87 & $13.00 \%$ & $100 \%$ \\
\hline Card number & 452 & $67.56 \%$ & $100 \%$ \\
\hline Date & 495 & $73.99 \%$ & $100 \%$ \\
\hline Weight & 0 & $0 \%$ & $100 \%$ \\
\hline
\end{tabular}

\section{RESULTS AND DISCUSSION}

\section{Completeness of Prescription}

Out of the 669 prescriptions $667(99.70 \%)$ had the name of the patient, $619(92.52 \%)$ had age of the patient and $87(13.00 \%)$ of prescriptions had diagnosis of the patient. However, none of the prescription had address and weight of the patient (table 1).

\section{Drug Related Information}

From the total of 1099 drugs issued, the correct name and strength of the drug were clearly stated in $800(72.79 \%)$ and 
Indo Global Journal of Pharmaceutical Sciences, 2020; 10(4): 39-45

$900(81.89 \%)$ of the prescriptions respectively. Quantity of the medication was rarely stated correctly in the prescription $36.94 \%$ (Fig.1).

Table 2: Summary of Prescription Paper Issued With Prescriber And dispenser related information at FHRH from January 10, 2019-May 10, 2019

\begin{tabular}{|c|c|c|c|c|c|}
\hline \multicolumn{2}{|c|}{ Prescriber information } & \multicolumn{2}{c|}{ Dispenser } & \\
\hline Parameters & Number & Percentage & Number(n) & Percentage & Gold \\
\hline Name & 219 & $32.74 \%$ & 140 & $20.93 \%$ & $100 \%$ \\
\hline Signature & 454 & $67.86 \%$ & 247 & $36.92 \%$ & $100 \%$ \\
\hline
\end{tabular}

Table 3: Summary of the prescribing practices at FHRH January 10, 2019-May 10, 2019

\begin{tabular}{|c|c|c|c|}
\hline \multicolumn{4}{|c|}{ Total number of prescription=669 } \\
\hline \multicolumn{4}{|c|}{ No of drugs prescribed $=1099$} \\
\hline Parameters & Frequency & Percentage & Gold \\
\hline $\begin{array}{c}\text { Average No of } \\
\text { drugs / } \\
\text { Prescription }\end{array}$ & 1.64 & & $<2$ \\
\hline Number of antibiotics & 295 & $43.94 \%$ & $<25 \%$ \\
\hline Number of injection & 8 & 11.95 & $<13$ \\
\hline Number of generic & 620 & $92.97 \%$ & $100 \%$ \\
\hline Number of EDL & 1099 & $100 \%$ & $100 \%$ \\
\hline
\end{tabular}

Table 4: Summary of patient care indicators at FHRH from January 10, 2019-May 10, 2019

\begin{tabular}{|c|c|c|c|}
\hline Indicator studied & \multicolumn{2}{|c|}{\begin{tabular}{|l|l|} 
Number Percentage \\
\end{tabular}} & WHO \\
\hline \multicolumn{4}{|l|}{ Interviewed patients $=669$} \\
\hline No .of drugs prescribed & 147 & $100 \%$ & $100 \%$ \\
\hline No. of drugs actually dispensed & 145 & $98.64 \%$ & $100 \%$ \\
\hline No .of drugs adequately labeled & 100 & $68.03 \%$ & $100 \%$ \\
\hline \multicolumn{4}{|l|}{$\begin{array}{c}\text { Educational status of } \\
\text { respondents }=669\end{array}$} \\
\hline $\begin{array}{c}\text { No of patients not able to read and } \\
\text { write }\end{array}$ & 253 & $37.81 \%$ & \\
\hline $\begin{array}{l}\text { Number of patients who attend } \\
\text { primary school }\end{array}$ & 70 & $10.46 \%$ & \\
\hline $\begin{array}{c}\text { Number of patients who attend } \\
\text { secondary school }\end{array}$ & 346 & $51.72 \%$ & \\
\hline
\end{tabular}

\section{Prescriber and Dispenser Related Information}

In this study, from the total of 669 prescription paper, $219(32.74 \%)$ had the name of prescriber and $140(20.93 \%)$ had the name of pharmacist/dispenser (table 2).
Prescribing Indicators

Out of 1099 drugs issued in 669 prescriptions, 43.94 of the prescription had antibiotics; about $12 \%$ had injectable drugs. Majority of the prescription issued with generic name of the drug and average number of drugs per prescription was 1.64 (table 3)

\begin{tabular}{|l|l|l|l|}
\hline $\begin{array}{l}\text { Knowledge status of the patient towards dispensed drugs, } \\
\text { n=669 }\end{array}$ & Frequency & Percentage & $\begin{array}{l}\text { WHO } \\
\text { standard }\end{array}$ \\
\hline Indicators & 112 & 16.74 & $100 \%$ \\
\hline Name of the drug & 639 & 95.5 & $100 \%$ \\
\hline $\begin{array}{l}\text { Frequency } \\
\text { administration }\end{array}$ & $\begin{array}{l}\text { Duration } \\
\text { administration }\end{array}$ & 698.95 & $100 \%$ \\
\hline Drug indication & 401 & 59.94 & $100 \%$ \\
\hline Precaution of drug & 164 & 24.5 & $100 \%$ \\
\hline Dose of the drug & 580 & 86.69 & $100 \%$ \\
\hline Patient satisfaction & 617 & 92.2 & \\
\hline Satisfied & 52 & 7.8 & \\
\hline Not satisfied & 52 & \\
\hline
\end{tabular}

Table 5: Summary of health facility indicators, at FHRH from January 10, 2019-May 10, 2019

\begin{tabular}{|c|c|c|}
\hline Items & $\begin{array}{c}\text { Availabl } \\
\text { e }\end{array}$ & $\begin{array}{c}\text { WHO } \\
\text { standar } \\
\mathbf{d}\end{array}$ \\
\hline Essential drug list & Yes & Yes \\
\hline Formulary2013 & Yes & Yes \\
\hline Standard treatmentguideline2014 & Yes & Yes \\
\hline TB treatment guideline & Yes & Yes \\
\hline HIV/AIDS treatment guide line & Yes & Yes \\
\hline National tracer drugs & & \\
\hline Amoxicillin tablets & Yes & Yes \\
\hline Oral rehydration salt & Yes & Yes \\
\hline Artemether/lumefantrine tablets & Yes & Yes \\
\hline Paracetamol tablets & Yes & Yes \\
\hline Mebendazole tablets & Yes & Yes \\
\hline TTC eye ointment & Yes & Yes \\
\hline Refampicin+isonizide+pyrazinamide+Etham & Yes & Yes \\
\hline Medroxyprogesterone(depo) injection & Yes & Yes \\
\hline Ergometrineinjection & Yes & Yes \\
\hline Ferrousgluconate tablets & Yes & Yes \\
\hline Pentavalant DPT-hep-Hib vaccine & Yes & Yes \\
\hline Zinc sulphatetablets & No & Yes \\
\hline Gentamycin injection & Yes & Yes \\
\hline
\end{tabular}

TB: Tuberculosis, HIV: immune deficiency virus, AIDS: acquired immune deficiency syndrome 


\section{Indo Global Journal of Pharmaceutical Sciences, 2020; 10(4): 39-45}

\section{Patient Care Indicators}

In this study 669 patients were included. Of which $230(34.37 \%)$ were females. Two hundred fifty three (37.81\%) of patients were illiterates (unable to read and write). From the total respondents, 595(88.93\%) of patients were able to repeat the correct dosage schedule of the drugs they had receive and 617(92.2\%) were satisfied by outpatient pharmacy service of the hospital. Regarding patient knowledge, $95.5 \%$ of patients knew about the frequency of their drug and only $16.74 \%$ of patients knew the name of drugs they received (table 4).

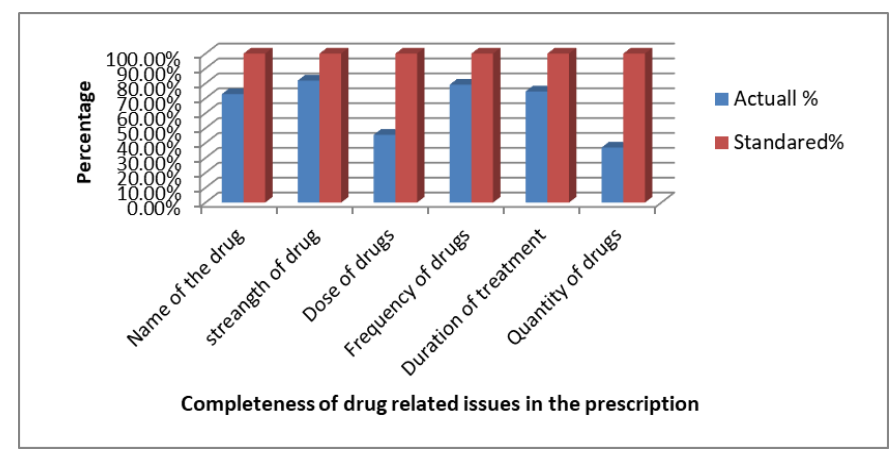

Fig.1: Summary of prescription paper issued with drug related information FHRH from January 10, 2019-May 10,2019

\section{Health Facility Indicators}

Availability of drug formulary, standard treatment guideline and tracer drugs

The purpose of assessing the availability of formularies and standard treatment guidelines is to examine how many numbers of this material is available. Formularies and standard treatment guidelines were available. From fifteen essential drugs, $90 \%$ of the key drugs were available. From thirteen types of national tracer drugs, $92.31 \%$ of them were available in this hospital (table 5)

A prescription is an important therapeutic transaction between the prescriber and drug consumer through dispenser. Complete prescription should include name, level of health institution and the main information: patient related information (name, card number, address, sex, age and diagnosis), drug related information (name, strength, dosage form, frequency and duration of treatment), and prescriber and dispenser name, and signature [10]. But in this study, most of the prescriptions lack most of the above information either drug related or patient related or both. This incomplete prescription may lead to patient harm or health professionals are unable to identify drug related problems previously happened.

Patient related information is included on the prescription and used to identify patients. In our finding, almost all of prescription had the name of the patient. Our study is higher than study done in Saudi Arabia but lower than Gondar university hospital (GUH) and Debremarkos referral hospital (DRH) $100 \%[11,12,13]$. This discrepancy might be because of time of study variation since the awareness of prescribers are increased from time to time and in GUH which is will organized university hospital. According to WHO, all prescription must contain the full name of the patients. Patient diagnosis should be written in every prescription to identify whether the drug is suitable for the patient or not and to identify drug disease interaction during dispensing by the pharmacist [14]. However, in our study only few of the prescription ordered with identified diagnosis. This result is lower than what was done in DRH. According to WHO, diagnosis should be written in all prescriptions [10].

It is apparent that rational selections of drugs dose and dosage forms would be highly determined by the age of the patient .The dose that should be administered to children would naturally be different from those given to adults, since age plays an important role in a successful management of the therapy [15].

If the pharmacists dispenses the drug without asking the age of the patient, he/she might wrongly dispense an adult dose to a child and vice versa, hence causing in either a therapeutic failure or over dose toxicity. The result is an indication that some prescribers need to be reminded of the scientific rational behind dose optimization versus patient age. In our study the age of the patient is written in the prescription in most of the prescription which is higher than the study done in Saudi Arabia and GUH but lower than DRH $[16,17,12]$.

The difference might be because in DRH, auditable pharmaceutical transaction and service which recommends highly for the completeness of prescription. This result is generally lower than the WHO [9] recommendation. Sex of the patients should also be specified on all prescriptions as some medicines could have sex dependent pharmacokinetic profiles [18]. In our study, most of prescriptions had sex which is higher than Saudi Arabia [16] and GUH [12] but lower than DRH [17]. This is also lower than the WHO [9] standard and we have to improve the prescription completeness.

Weight of the patients should be specified on all prescription some medicines which have both pharmacokinetics and pharmacodynamics profiles depend on weight of the patient. Although the concept of varying the dose with the body weight has long tradition, adult doses have been as summed to be the same irrespective of the size or shape [15]. The result is 


\section{Indo Global Journal of Pharmaceutical Sciences, 2020; 10(4): 39-45}

an indication that some prescribers need to be reminded of the scientific rational behind dose optimization versus patient weight. According to WHO [9], all prescription should have been present the weight of the patient. Medication dose calculated based on weight especially for children's. So thus data needs to be improved as part of promoting the rational drug prescribing and the right dose of dispensing to prevent therapeutic failure and toxicity.

In our study from the total drugs dispensed, the correct name and strength of the drug were clearly stated in more than half of the prescriptions but still there were prescriptions which were incomplete. In most prescriptions physician wrote the name of the drug in abbreviation. In this study still there is a gap in dose, frequency and duration of treatment were not clearly stated in the prescription which may lead to confusion for the health professionals and patients and contribute for irrational drug use. This result also lowers than study done in DRH [17]. This discrepancy might be in DRH, there was practice of electronic dispensing system.

A prescriber is not always a medical doctor; he/she can be paramedical workers such as a medical assistant, midwife, or orders drug(s) to patients. Therefore, in order to identify the person involved in prescribing, the prescription should include the prescriber's name, qualification, signature and the date on which the prescription was written because there is a limit after which the prescription will not issue. Prescriber's name and address will allow either the patient or the dispenser to contact the prescriber for any clarification or potential problem with the prescription [10]. In our study, the name of the prescriber was not well written on some of the prescriptions posing the issue of accountability in the medico legal issue. This information is important for cross checking in case of prescriptions errors and help in easy identification of the liable prescriber. In our finding, only less than half of the prescription dispensed with the name of prescriber and slightly greater than half dispensed with the signature of physicians. This study is almost similar with the study done in GUH [12]. The name of prescriber was not wrote well and unreadable which may contribute for irrational drug use.

Dispensers are not always a pharmacist but also can be a pharmacy technician and an assistant who is licensed to or authorized to dispense drugs. It is the primary responsibility of the pharmacist to assure the correct dispensing of the drug and maintaining the quality of the drug dispensed. Dispensing error may be common when the dispensing is performed low level of health care provider. Therefore, information which used to identify the dispenser who issued the drug to the patient (name and signature) on the prescription should be briefly recorded. In this study only less than half of the prescription contained both dispenser name and signature which is very low as compared to study done in GUH [12]. So this study showed that since dispenser information is crucial to combat irrational drug use through communication with the prescribers and patients.

Prescribing multiple drugs to patients at once (poly-pharmacy) it may lead missing of dose, over dosing and drug-drug interaction or drug food interaction and the patient may be fatigue. The average number of drugs prescribed per prescription was 1.64. This result is within WHO recommendation and lowers than study done in Nepal, China, India, DRH and GUH [19, 16, 20, 17, 12]. Regarding generic prescription, in our study, most of the drugs prescribed with generic name and this result is better than what was done in Nepal, India and Saudi Arabia[19, 20 16]. However, it is less than what is recommended in WHO [17]. Therefore, it needs further intervention to achieve the standard.

Antibiotics consumption in this hospital is beyond recommended by WHO $(<25 \%)$ in which near to half the prescriptions issued antibiotics. However, this result is greater than what was reported from China, Nepal, India and GUH $[16,19,20,12]$. So this showed that the hospital should try to prepare antibiotics stewardship and prevent antibiotics resistance. It also contributes for patient harm in terms of poor patient outcomes and adverse drug reactions. Regarding with percentage of injectable drugs, only less than a unit was injectable prescription which is lower than as compared to previous study in Nepal, China, Hawassa, DRH and Gondar $[19,16,21,17,12]$. This result is within the recommended WHO standards. All the drugs were prescribed from hospital essential drug list (EDL) which is in line with WHO [21] standard in which all $100 \%$ of prescribed medication should be under essential drug list.

In our finding, all drugs prescribed from the hospital EDL. WHO (9) recommended that $100 \%$ of drugs should be prescribed from EDL. This is in line with WHO recommendation and Debremarkos. However, it is better than study done in Nepal, China, India, Hawassa and GUH [19, 16, $20,21,12]$.

In our study, the average time spent for counseling was 11.54 min. which is within WHO [21] recommendation but less than what was done in Nepal [19]. This discrepancy might be the presence of qualified health professionals and spent more time on discussion with their patient. More than half of dispensed drugs were labeled and it is better than in Nepal, India and GUH [19, 20, 12] but it is still below WHO [17] recommendation in which all dispensed drugs should be 
Indo Global Journal of Pharmaceutical Sciences, 2020; 10(4): 39-45

labeled. It is important to uniquely identify the contents of the container and to ensure that the patient have clear and concise information about the use of the drug. In this hospital most of the prescribed drugs were actually dispensed and which is better than what was done in India and Gondar but it does not meet WHO [9] standards actually all drugs prescribed should be dispensed the hospital.

Limitation: this drug use evaluation was conducted only in single center.

\section{CONCLUSION}

From this results, it can be concluded that almost all prescriptions were incomplete and lack the necessary patient related information(sex, age, weight, patient diagnosis)and drug related information(correct name, strength, dose, frequency, duration, quantity of the drug) and also the name and signature of the prescriber and dispenser. With regard to the WHO limit of generic and antibiotic use at FHRH fails to maintain the limit, so it needs some improvement on generic and antibiotics use pattern in the hospital. Almost all drugs available in the hospital but labeling on each and every dispensed drug to patients and also both good dispensing and counseling practice are low. Therefore, good labeling, dispensing and counseling practice are required to upgrade patient knowledge and adherence to treatment. The good thing found in this hospital is there is availability of tracer drugs.

\section{ABBREVIATIONS}

DRH: Debremarkos referral hospital; EDL: Essential drug list; FHRH: Felege hiwot referral hospital; GUH: Gondar university hospital; WHO: World health organization

\section{ETHICAL CONSIDERATIONS}

Ethical approval was secured from hospital drug and therapeutic committee. Before data collection permission from head of hospital pharmacy was obtained. Verbal consent from patients was secured to extract data from patents'. Privacy and confidentiality were ensured during patient interview.

\section{ACKNOWLEDGEMENT}

I am very grateful to data collectors. I would like to give special thank you for my study participants for their cooperation to participate for this study. I would like to also appreciate peoples formally or informally help me to accomplish this study.

\section{DATA AVAILABILITY}

All data analyzed during this study was available for publication.

\section{CONFLICTS OF INTEREST}

The authors declare that they have no competing interests.

\section{FUNDING SOURCE}

No external funding declared.

\section{AUTHOR'S CONTRIBUTIONS}

Bezie Kebede: proposal development, data analysis and report writing. Biset Asredaw: conceptualization and methodology

\section{REFERENCES}

1. The Rational Use of Drugs. Report of a conference of experts,Nairobi,25-29 November1985 .Geneva: World Health Organization; 1987.

2. Food, Medicine and Healthcare Administration and Control Authority (FMHACA) of Ethiopia. 2nd edition. Addis Ababa: Manual for Medicines Good Prescribing Practice; 2012.

3. WHO:WHO Medicines Strategy: Frame work for action Essential drugs and Medicines policy 2000 - 2003 .Geneva: WHO; 2000. (WHO/EDM/2000.1).

4. WHO: The use of Essential Drugs.6thReport of the Expert Committee. Geneva: WHO Technical report Series 850.3; 1995.

5. HogerzeilHV,WalkerGJA,SallamiAO,FernandoG:Impactofaness entialdrugsprogram on availability and rational use of drugs

6. Chukwuani, CM. Onifade, M., Sumonu,K.Survey of drugs use practice and antibiotics prescribing pattern at general hospital in Nigeria. Pharm world Sci 2002;24 (5): 188-195

7. The Drug Administration and Control Authority (DACA) of Ethiopia. Zonal Hospital Standard Treatment Guideline 2010.xixii24

8. Laing RO, Hogerzeil HV: Ten recommend to improve use of medicines in developing countries. Health Policy Plan 2001, 16(1):13-20.

9. WHO. Action Program on Essential drugs, How to investigate drug use in health facilities. World health organization, Geneva1993: 1-87

10. Drug Administration and Control Authority of Ethiopia.Standardtreatmentguidelinesfor general Hospitals: DACA; January2010, xvi-xvii.

11. Decosta,A.,Bhartiya,S.,N and eswar,S.,Diwan,VK. Patterns of drug in the public sector primary health centers of Bhopal district.Pharm.World Sci. 2008, 30 (6): 684-693.

12. Admassie E,et al: Assessment of Drug Use Practices and Completeness of prescriptions in Gondar University teaching referral hospital. Int J Pharm SciRes. 2013;4(1); 265-275.

13. HogerzeilHV, Bimo, Ross-DegnanD, Laing RO, Ofori-AdjeiD, SantosoB, Azad ChowdhuryAK, DasAM, KafleKK, MabadejeAF: Field tests for rational drug use in twelve developing countries. Lancet, 1993;342(8884):14081410.

14. Zou, J., Li ,L., Zhang, C., Yan, Y., Gao, F., and Zhang, H. Analysis of outpatient prescription indicators and trends in Chinese Jingzhou area between September1and10,2006-2009. African Journal of Pharmacy and Pharmacology2011, (2), 270275,

15. Chukwuani,CM., Onifade,M., Sumonu,K. Survey of drugs use practice and antibiotics prescribing pattern at general hospital in Nigeria. Pharm world Sci 2002;24 (5): 188-195. 
Indo Global Journal of Pharmaceutical Sciences, 2020; 10(4): 39-45

16. HengWang, NiannianLi etal: Prescription Pattern and Its Influencing Factors in Chinese County Hospitals: retrospective Cross-Sectional studyDOI:10.1371/ journal.pone.0063225.

17. WHO: How to investigate drug use in health facilities: selected drug use indicators. Geneva: WHO/DAP/93.1;1993WHO.How to investigate drug use in health facilities: selected drug use indicators, Action Program on Essential Drugs 1993, WHO/DAP/93.1.

18. Bimo: Field testing of drug use indicators of INRUD: report of field trip to Indonesia Bangladesh, and Nepal, June-July1991.In How to investigate drug use in health Facilities .WHO:Geneva;1993.74(WHO/DAP/93.1).

19. Suni,K.,Punam,S.,Madhuri et al. Pattern of prescription and drug dispensing in Lokmanya Medical College and General hospital, Mumbai, India. Indian Journal of Medicine,2005:vol72,No2:117-121.

20. Anteneh Assefa Desaleg et al. Completeness of prescription and rational drug use, Hawassa, Ethiopia, BMC Health Services Research, 2013, 13:170 doi:10.1186/1472-6963-13-170.

21. WHO: World Health Organization promoting rational use of medicines: core components.

Indo Global Journal of Pharmaceutical Sciences( ISSN 2249 1023; CODEN- IGJPAI; NLM ID: 101610675) indexed and abstracted in CrossRef (DOI Enabling), CNKI, UGC CARE Journal List, EMBASE (Elsevier), National Library of Medicine (NLM) Catalog (NCBI), ResearchGate, Publons (Clarivate Analytics), CAS (ACS), Index Copernicus, Google Scholar and many more. For further details, visit http://iglobaljournal.com 